III. Erklärung der diamagnetischen Wirkungsweise durch die Ampère'sche Theorie;

con Dr. o. Feilitzsch,

Prof. in Greifswald.

( 7 weite Abhandlang, die erste in d. Ann. Bd. 87, S. 209 u. 427.)

Diamagnetismus und Magnetismus entspringen aus einer identischen Erregung der kleinsten Massentheilchen.

1. Das Material zu der nachfolgenden zweiten Abhandlung der "Erklärung diamagnetischer Wirkungsweise durch die Ampere'sche Theorie "lag bereit, als die erste $\Lambda$ bhandlung in Poggendorff's Annalen Bd. LXXXVII, S. 206 und 427 erschienen war. Mittlerweile hatte $\mathrm{Hr}$. W eber dic dritte Abtheilung seiner "elek trodyuamischen Maafsbestimmungen, insbesondere über den Magnetismus “ veröffentlicht. Da die darimnen aufgestellte Theorie, gestützt auf die simreichsten Versuche, gerade das Gegentheil von dem aussagte, was $u$. a. in jener meiner ersten Abhandlung bewiesen werden sollte, so war mir eine Wiederholung und weitere Fortführung jener Versuche zur Pflicht gemacht, um entweder mich ihren Consequenzen anzuschliefsen, oder um die von mir ausgesprochenen Ansichten stärker zu befestigenl.

2. Meine bisherigen Untersuchungen hatten mich zu folgenden Resultaten gefuhrt:

Der eisemmagnetische, der diamagnetische und der sauerstoffmagnetische Zustand entstchen aus derselben und in allen drei Zuständen gleichgerichteten Polarität der kleinsten Theilchen.

Diese drei Zustände unterscheiden sich nur 1) durch die Entfernung der kleinsten Theilchen voneinander und die dadurch verninderte magnetische Molecularinduction und 2) durch die Art, wie in denselben die maguetische Polarität erreğt wird. 
Die relative Entfernung (und Gröfse) der kleinsten Theilchen bedingt den Unterschied zwischen den eisenmagnetischen, wismuthmagnetischen und sauerstoffmagnetischen Körpern. Je näber die kleinsten Theilchen, bei sonst gleicher Gröfse, aneinander liegen, desto mehr überwiegt die magnetische Molecularinduction über die Wirkung der äufseren magnetischen Induction. Die erste Körperklasse ist durch die geringste, die letzte durch die gröfste relative Entfernung der kleinsten Theilchen charakterisirt.

Erfährt ein länglicher Körper in der Mitte seiner Längs ausdehnung (etwa durch eine galvanische Spirale von geringerer Länge als der Körper) eine stärkere äufsere magnetische Induction als an den Enden, so wird er, welche der drei Klassen er auch angehören mag, stets in den eisenmagnetischen Zustand versetzt.

Dahiugegen treten die drei genannten Zustände an den drei verschiedenen Körperklassen' auf, wenn dieselben in länglicher Form von den Enden her stärker magnetisch inducirt werden, als von der Mitte der Längsausdehnung (etwa durch Aufhängen zwischen zwei Magnetpolen).

Nach der Richtung der magnetischen Axe ist im magnetischen Zustand das magnetische Moment in der Mitte der Axe grölser als an den Enden derselben; im diamagnetischen und sauerstoffmagnetischen Zustand dagegen ist es an den Enden gröfser als in der Mitte der Axe.

Nach der Richtung des Querschnitts (senkrecht zur magnetischen Axe) findet dagegen folgende Vertheilung der magnetischen Momente statt: in eisenmagnetischen Körpern ist dasselbe in der Mitte $=0$ und ist an der Peripherie und in geringem Abstand von derselben von gleicher Gröfse, in wismuthmagnetischen Körpern nimmt das magnetische Moment von der Peripherie nach der Mitte $a b$, ohne $=0$ zu werden, in sauerstoffmagnetischen Körpern endlich besitzt das mag- 
netische Moment über den ganzen Querschnitt hinweg denselben Werth ').

Ein wismuthmagnetischer Körper flieht vor dem erregenden Pole und ein magnetischer wendet sich ihı $\mathrm{zu}$, wesentlich deswegen weil sie das Bestreben haben von Stellen schwächerer Magnetkraft zu Stellen stärkerer Magnetkraft überzugehen. Und zwar liegen die Stellen der durch einen äufsern Pol in einem eisenmagnetischen oder wismuthmaguetischen Querschnitt erregten grö/sten Magnetkraft in beträchtlichem Abstand von diesem Querschnitt, während sie bei einer sauerstoffmaguetischen Substanz in dem Querschnitt selbst liegen.

Daraus folgt, dafs ein jeder sauerstoffmagnetische Querschnitt, folglich auch der ganze sauerstoffnagnetische Körper das Bestreben hat, sich den erregenden Magnetpolen anzunăhern. Ferner mufs, wegen der magnetischen Vertheilung in der Richtung der Axe die Stelle stärkster Magnetkraft eines länglichen eisenmagnetischen Körpers in dem Körper selbst liegen, derselbe also ebenfalls von einem äufsern Magnetpole angezogen werden. Dagegen aber mufs die Stelle stärkster Magnetkraft eiues länglichen wismuthmagnetischen Körpers wegen der Vertheilung in der Richtung der Axe aufserhalb und in beträchtlicher Entfernung vom Körper liegen, weswegen er das Bestreben hat, vor dem erregenden Magnetpol zu fliehen.

3. Dahingegen leugnet $\mathrm{Hr}$. Weber die gleiche Polarität der klcinsten Theilchen bei eisenmagnetischen und wis-

1) Zur bequemeren Uebersicht sind die Behauptungen dieses und des vorigen Satzes in Fig. 3 Taf. HI bildlich dargestellt. Daselbst bedeuten $\boldsymbol{N}$ und $\boldsymbol{S}$ die erregenden äufseren Magnetpole, $a$ einen eisenmagnetischen, $b$ einen wismuthmagnetischen und $c$ cinen sauerstolfmagnetisclien Körper. Durch ns ist die Riclıtung der magnetischen Polaritüt der kleinsten Theilchen angedeutet; die Ordinaten der Curve über der Axe ox entsprechen dem magnetischen Moment der resp. Schichten in der Richtung der magnetischen Axe, und die Ordinaten der Curve über der Axe oz dem magnetischen Mument der resp. Theilchen des Querschnits. 
wismuthmagnetischen Körpern und erklärt das diamagnetische Verhalten zum Unterschied von dem magnetischen daraus, dafs ein erregender Pol in den kleinsten Theilchen wismuthmagnettscher Körper den gleichnamigen Pol sich zuwende und den ungleichnamigen abstofse, während in den kleinsten Theilchen der magnetischen Körper ein erregender Pol sich den ungleichnamigen Pol zuwende und den gleichnamigen zurückstofse. Wenn ich recht sehe, soll aber die diamagnetische Polarität nicht eigentlich ihren Urgrund in einer Scheidung der beiden entgegengesetzten Maguetismen der kleinsten Theilchen (umgekehrt wie bei magnetischen Körpern) haben, sondern darin, dafs durch die Aunäherung eines Magneten oder einer galvanischen Spirale Molecularströme inducirt werden, welche deu momentanen Inductionsströmen gleichgerichtet sind und somit gleichwerthig einer der magnetischen entgegengesetzten Polarität. Diese Molecularinductionsströme sind der magnetischen Scheidungskraft ciner benachbarten erregenden Ursache proportional, und da sie ohne Widerstand un die Molecule kreisen, dauern sie ungeschwächt fort, bis die erregende Ursache sich entfernt und dadurch sie vernichtet. Die in gröfseren Babnen sich um einen Leiter bewegenden Inductionsströme werden dagegen durch denjenigen Widerstand gleich nach ihrem Entstehen vernichtet, den dieselben in dem Leiter vorfinden.

4. Das aber cine der magnetischen entgegengesetzte Polarităt oder etwas derselben Gleichwerthiges den diamag. netischen Zustand begründc, beweist Hr. Weber durch folgenden an der Spitze der genannten Untersuchungen stehenden Fundamentalversuch: Ein Hufeisenmagnet $N S$ (Poggendorff's Annalen LXXXVII, Tafel III u. S. 168 Fig. 2. 3. 4.) ist an einem Faden aufgehangen, so dafs beide Schenkel in derselben Horizontalebene schwingen können. Zwischen beiden Schenkeln befindet sich die Mitte einer vertical stehenden langen galsanischen Spirale, innerhalb welcher ein Cylinder vou chemisch reinem Wismuth $a a$

Poggendorf's Annal. Bd. XCII. 
und von weniger als der halben Läuge der Spirale mittelst eines Fadens aus der Ferne auf und abbewegt werden kann. Bei dieser Bewegung befindet sich abwechselnd das untere und das obere Ende des Cylinders zwischen den Magnetpolen. In Folge des Zustandes, in welchen das Wismuth durch die thätige galvanische Spirale versetzt wird, wirkt dasselbe in beiden Lagen entgegengesetzt ablenkend auf den Magneten. Diese Ablenkung wird, wie am Magnetometer, durch Fernrohr, Scale und Spiegel beobachtet, welcher letztere $P$ an der Drehungsaxe des Magneten kefestigt ist. Befinden sich die Schenkel des Magneten in einer Horizontalebene, welche gerade die verticale Spirale halbirt, so dürfte dieselbe eigentlich keine Ablenkung auf den Magneten ausüben. Um aber etwaige geringe Ablenkungen (die namentlich aus dem Eisengehalt des Kupferdrahtes hervorgehen) zu compensiren, wird in einiger Entfernung vom Magneten ein Multiplicator $M$ von wenigen Windungen anfgestellt und durch den Strom der Spirale in Thätigkeit versetzt. Bringt man nun vom Fernrohr aus durch die gewöhnlichen Mittel den schwebenden Hufeisenmagneten in Ruhe und hebt alsdann den Wismuthstab innerhalb der Spirale, so dafs sein unteres Ende in die Ebene der Pole desselben kommt, so beobachtet man eine Ablenkung durch Verrückung des Skalenbildes am Verticalfaden des Fernrohrs. Die dadurch veranlafste Schwingung des Magneten wird vermehrt, wenn man beim Rückgang desselben den Wismuthstab wieder senkt. Durch abwechselndes Heben und Senken des Stabes im Takte der Schwingungen des Magneten kann man diesem beträchtliche Ausschlägge ertheilen und im umge. kehrten Takte diese Ausschläge wieder bis zur Ruhelage vermindern.

Wird statt des Wismuthstabes ein Eisenstab (Draht) von derselben Länge, aber von möglicht geringer Dicke, in derselben Weise auf und abbewegt, so zeigt sich, dafs der Hufeisenmagnet die entgegengesetzte Ablenkung erhält, also z. B. beim Heben höhere Scalentheile ins Fernrohr 
führt, während der Wismuthstab beim Heben eine Bewegung zu niederen Scalentheilen veranlafst.

5. Aus diesen Versuchen schliefst Hr. We ber:

dafs gleiche Scheidungskraft entgegengesetzte ideale Vertheilungen beim Eisen und beim Wismuth hervorbringt, oder umgekehrt, dafs eine gleiche ideale Vertheilung bei Eisen und Wismuth entgegengesetzt gerichteten Scheidungskräften entspricht ').

6. Diese Versuche wiederholte ich mit Apparaten von beträchtlich grölseren Dimensionen, als die sind, welche Weber zu seinen Versuchen benutzte. Die hier und im Folgenden gebrauchten Vorrichtungen mufsten soweit als immer möglich von mir selbst construirt werden, wodurch ich am sichersten ein Urtheil über die Gränze ihrer Brauchbarkeit erbielt. Die galvanische Spirale war auf einen Pappcylinder von 520 ${ }^{\mathrm{mm}}$ Länge, $3^{\mathrm{mm}}$ innerem Durchmesser und $4^{\mathrm{mm}}$ Pappdicke aufgewunden. Sie bestand aus Kupferdraht von etwas mehr als $1^{\text {mm }}$ Durchmesser, der mit Seide umsponnen und zur besseren Isolation zu wiederholten Malen mit Bernsteinlack getränkt war. Das Gewicht des Drahtes betrug mit der Ueberspinnung etwa $2^{\mathrm{kgr}}, 2$ und diese waren auf 4 Lagen von $510^{\mathrm{mm}}$ Länge und je 305 Windungen vertheilt. Jede Lage wurde von der anderen durch zwei Lagen starker Seide getrennt, welche mit Schellacklösung mehrfach gefirnifst war. Der Magnet NS (Pogg. Ann. Bd. 87, Heft III. Fig. 2. 3. 4.) bestand aus einem Stück Gufsstahl von $330^{\mathrm{mn}}$ Länge und $18^{\mathrm{mm}}$ Seite, welches hufeisenförmig gebogen war, so dafs die Pole im lichten $73^{\mathrm{mm}}$ von einander abstandeo. Befand sich zwischen beiden Schenkeln die Spirale, so stand jeder noch $6^{\mathrm{mm}}, 5$ von derselben ab. Am Magneten war der Spiegel $P$ befestigt, und beide hingen an 15 Coconfäden von $2^{\mathrm{m}}, 4$ Länge von der Decke des Zimmers herab. Der Wismuthstab $a a$ von chemisch - reinem Wismuth (aus der Apotheke zum Einhorn in Berlin) war $248^{\mathrm{mm}}$ lang, hatte $20^{\mathrm{mm}}$ Durch-

1) Elektrodynamische Maafsbestimmungen S. 541. Pogg. Ann. Bd. 87, S. 147. 
messer und wog 778 ${ }^{g r}, 8$. Bei der höclssten Hebung und tiefsten Senkung innerhalb der Spirale standen seive Enden noch wenigstens $16^{\mathrm{mm}}$ von den resp. Enden der Spirale ab. Dic Rolle, an welcher der Wismuthstab aufund abbewegt wurde, war ebenfalls an der Decke des Zimmers befestigt. Fernrohr und Scale waren in etwa $2^{\mathrm{m}}, \mathbf{5}$ Abstand vom Spiegel des Magneten aufgestellt. Da ich keine messenden Versuche beabsichtigte, unterliefs ich es den Magneten mit einem Dämpfer zu umgeben.

7. Versuch 8. Die Spirale wurde mit 6 Grove'schen Elementen von je 80 Quadratcentim. Platinblech in Thätigkeit versetzt. Der Nordpol der Spirale war nach oben gekehrt, und der Nordpol des Magneten, sowie die höheren Scalentheile befanden sich zur Linken des hinter dem Fernrohr stehenden Beobachters. Nachdem der Magnet so beruhigt war, dafs er nur noch sehr kleine Schwingungen vollführte, wurde der Wismuthstab in der Spirale gehoben und der grölste Ausschlag nach rechts (in Fernrohr nach links) in Zehnteln von Millimetern der Scale beobachtet und notirt. Alsdann wurde der Stab wieder gesenkt und wiederum der gröfste Ausschlag nach links notirt. So wurde abwechselnd der Stab sieben Mal gehoben und gesenkt. Hierauf blieb der Stab während einer einfachen Schwingung in der unteren Lage, und wurde dann wieder sieben Mal in Takt der Schwingungen gehoben und gesenkt. Demnächst wurde er während einer Schwingung in der oberen Lage gelassen, um wieder dasselbe Spiel zu vollführen $u$. s. f. In der nachfolgenden Tabelle sind unter der Ueberschrift, Wismuthcylinder, die so gewonnenen Resultate einer von viclen in dieser Weise angestellten Beobachtungsreihen aufgczählt. Und zwar ist in der ersten Columne die jedesmalige Stellung des Wis. muthstabes, in der zweiten der entsprechende gröfste Ausschlag des Magneten in Zehnteln von Scalentheilen und in der dritten die Differenz zwischen zwei aufeinanderfolgenden Ausschlägen, d. i. Schwingungsbogen angegeben. Die Zahlen unter der Ueberschrift "Wachscylinder " wer- 
den bei Gelegenheit des dritteu Versuchs No. 12 Erörterung finden.

\begin{tabular}{|c|c|c|c|c|c|c|c|c|}
\hline \multirow[b]{2}{*}{$\begin{array}{c}\text { Der } \\
\text { Cylinder } \\
\text { war }\end{array}$} & \multicolumn{4}{|c|}{$\begin{array}{l}\text { a) Versuche mit den VVisnuull. } \\
\text { cylinder. }\end{array}$} & \multicolumn{4}{|l|}{$6\rangle$} \\
\hline & $\begin{array}{c}\text { Beol- } \\
\text { ichlicte } \\
\text { Zialslen. }\end{array}$ & $\begin{array}{l}\text { Schwin- } \\
\text { gungs- } \\
\text { weite. }\end{array}$ & \begin{tabular}{|} 
Fortse \\
Beob- \\
achtete \\
Zahlen \\
\end{tabular} & $\begin{array}{l}\text { etzung. } \\
\text { Scliwin } \\
\text { gungs- } \\
\text { wcite. }\end{array}$ & $\begin{array}{l}\text { Beob- } \\
\text { achtete } \\
\text { Zalilen }\end{array}$ & $\begin{array}{l}\text { Schwin } \\
\text { gungs- } \\
\text { weile. }\end{array}$ & $\begin{array}{l}\text { Forts } \\
\text { Bcob- } \\
\text { aclitele } \\
\text { Zahlen. }\end{array}$ & $\begin{array}{l}\text { Setzung. } \\
\text { Scliwin. } \\
\text { gungs- } \\
\text { wacile. }\end{array}$ \\
\hline gesenkı & & & 5306 & & & & 5291 & \\
\hline gehoben & 5426 & & 5582 & 264 & 5250 & 249 & 540 & 101 \\
\hline gesenkt & 5492 & 89 & 5318 & 247 & 5499 & 258 & 5299 & 113 \\
\hline $\begin{array}{l}\text { gehoben } \\
\text { gesenkt }\end{array}$ & 5403 & 122 & 5565 & 240 & 5241 & 194 & 5412 & 133 \\
\hline $\begin{array}{l}\text { gesenkt } \\
\text { ehoben }\end{array}$ & $\begin{array}{r}5525 \\
5328\end{array}$ & 147 & $\begin{array}{r}5325 \\
5350\end{array}$ & 225 & $\begin{array}{r}5435 \\
5270\end{array}$ & 165 & $\begin{array}{r}5279 \\
5445\end{array}$ & 166 \\
\hline $\begin{array}{l}\text { gelioben } \\
\text { gesenkt }\end{array}$ & $\begin{array}{r}5368 \\
5545\end{array}$ & 167 & $\begin{array}{c}5301) \\
5340\end{array}$ & 210 & $\begin{array}{r}5210 \\
54.30\end{array}$ & 160 & $\begin{array}{c}5443 \\
5248\end{array}$ & 197 \\
\hline gehoben & 5358 & 187 & 5541 & 201 & 5275 & 155 & 5441 & 193 \\
\hline gesenkt & 5567 & 209 & 5355 & 186 & 5443 & 168 & 5250 & 191 \\
\hline hoben & 5340 & 7 & 5522 & 1 & 5258 & 185 & 5440 & $\begin{array}{l}190 \\
198\end{array}$ \\
\hline gesenkt & $\mathbf{5 5 8 3}$ & 213 & 5.379 & & 5430 & 172 & 5242 & \\
\hline thoben & 5319 & 2 & 5512 & $\begin{array}{l}100 \\
121\end{array}$ & 5270 & $\begin{array}{l}100 \\
172\end{array}$ & 542 & \\
\hline $\begin{array}{l}\text { nkt } \\
\text { en }\end{array}$ & $\begin{array}{r}5601 \\
5906\end{array}$ & 305 & $\begin{array}{r}5391 \\
5480\end{array}$ & 98 & 5442 & 174 & & 185 \\
\hline $\begin{array}{l}\text { ehoben } \\
\text { gesent }\end{array}$ & $\begin{array}{c}5296 \\
5621\end{array}$ & 325 & $\begin{array}{r}3489 \\
5412\end{array}$ & 77 & $\begin{array}{r}5268 \\
5420\end{array}$ & 152 & $\begin{array}{c}5335 \\
5258\end{array}$ & 177 \\
\hline & & 326 & & 77 & & 148 & & 174 \\
\hline $\begin{array}{l}\text { gesenkt } \\
\text { chuben }\end{array}$ & $\left|\begin{array}{r}5295 \\
5603\end{array}\right|$ & 308 & $\begin{array}{r}5489 \\
5384\end{array}$ & 105 & $\begin{array}{r}5272 \\
5423\end{array}$ & 151 & $\begin{array}{l}54332 \\
5262\end{array}$ & 170 \\
\hline $\begin{array}{l}\text { ghoben } \\
\text { gesenkt }\end{array}$ & $\begin{array}{r}5603 \\
5312\end{array}$ & 281 & $\begin{array}{r}53 \\
5\end{array}$ & 120 & $\begin{array}{c}5423 \\
5268\end{array}$ & 155 & $\begin{array}{r}3202 \\
5416\end{array}$ & 144 \\
\hline lioben & 5580 & 268 & $\begin{array}{l}530 \\
5: 368\end{array}$ & 136 & $\begin{array}{c}5200 \\
5420\end{array}$ & 152 & 5272 & 134 \\
\hline gesenkt & 5328 & $\begin{array}{l}252 \\
629\end{array}$ & 5523 & & 5275 & 145 & 5.389 & 117 \\
\hline lioben & 5557 & $\begin{array}{l}229 \\
212\end{array}$ & $\mathbf{5 3 5 \overline { 5 }}$ & & 5408 & 133 & 5285 & 104 \\
\hline gesenkt & 5345 & 205 & 538 & & 5285 & 12 & 5395 & 110 \\
\hline $\begin{array}{l}\text { ioben } \\
\text { gesenkt }\end{array}$ & 5540 & 173 & 5341 & 214 & 5390 & $\begin{array}{r}115 \\
90\end{array}$ & 5300 & \\
\hline $\begin{array}{l}\text { gesenkit } \\
\text { hoben }\end{array}$ & $\begin{array}{r}5367 \\
\end{array}$ & 153 & $\begin{array}{r}5555 \\
\end{array}$ & 238 & 5300 & 92 & 5389 & 11 \\
\hline $\begin{array}{l}\text { doben } \\
\text { gesenkt }\end{array}$ & 5320 & 138 & 5317 & 261 & 5392 & 116 & 5275 & 1. \\
\hline $\begin{array}{l}\text { gesenkt } \\
\text { hoben }\end{array}$ & 5382 & 116 & $=9$ & 272 & 5286 & 114 & $\begin{array}{r}5395 \\
\end{array}$ & 1 . \\
\hline $\begin{array}{l}\text { ehoben } \\
\text { gesenkt }\end{array}$ & $\begin{array}{c}5498 \\
5402\end{array}$ & 96 & $\begin{array}{c}5306 \\
5587\end{array}$ & 281 & $\begin{array}{c}5400 \\
5290\end{array}$ & 110 & $\begin{array}{r}5270 \\
5399\end{array} \mid$ & 129 \\
\hline gehobe & 5460 & & 5288 & & 5391 & 101 & 5280 & \\
\hline Hol & 5395 & & 5590 & & 5279 & 112 & 5420 & \\
\hline & 5500 & 119 & 5305 & & 5405 & 120 & 5273 & 147 \\
\hline Len & 5381 & 119 & 5569 & $\begin{array}{l}2 \\
2\end{array}$ & 5285 & 120 & 5425 & \\
\hline & 5518 & 156 & 5329 & 240 & 5392 & 97 & 5250 & \\
\hline gelioben & 5362 & 176 & 5550 & 205 & 5295 & 120 & 5113 & \\
\hline gesenkt & 5538 & 193 & $\mathbf{6 3 4 5}$ & 187 & 5415 & 127 & 5260 & $\begin{array}{l}153 \\
159\end{array}$ \\
\hline gehobed & $\mathbf{5 3 4 5}$ & 207 & 5532 & 177 & 5288 & 125 & 5419 & 168 \\
\hline Gesenkt & $\begin{array}{r}555 \\
-0.02\end{array}$ & 225 & 5355 & 164 & 54 & 139 & $\begin{array}{r}5251 \\
\end{array}$ & 19 \\
\hline gchoben & 5327 & 257 & $\begin{array}{r}5519 \\
534\end{array}$ & 139 & 5274 & 156 & 5445 & 21 : \\
\hline & & 284 & & 129 & $\begin{array}{r}5430 \\
5 \cdot 260\end{array}$ & 170 & $\begin{array}{r}5232 \\
5131\end{array}$ & 199 \\
\hline & 5300 & 302 & & 124 & 5260 & 195 & $\begin{array}{r}0431 \\
5241\end{array}$ & 190 \\
\hline & & 32 & 9485 & 100 & 5261 & 19.4 & 5430 & 18 \\
\hline$g \csc n k t$ & $\begin{array}{r}3200 \\
5618\end{array}$ & $3: 3$ & 5395 & 90 & 5420 & 16 & 5242 & 188 \\
\hline & & & & & & & & \\
\hline
\end{tabular}


Diese Zahlen zeigen nun so übereinstimmend und augenscheinlich als möglich, dafs der Magnet nach $\left\{\begin{array}{l}\text { niederen } \\ \text { löheren }\end{array}\right\}$ Scalentheilen getrieben wurde, wenn der Wismuthstab sich in $\operatorname{der}\left\{\begin{array}{l}\text { oberen } \\ \text { untercn }\end{array}\right\}$ Lage befand, dafs also der $\left\{\begin{array}{l}\text { Sïdpol } \\ \text { Nordpol }\end{array}\right\}$ des Magneten angezogen wurde, wenn sich zwischen seinen Schenkeln dasjenige Eude des Wismuthstabes befand, welches $\operatorname{dem}\left\{\begin{array}{l}\text { Siidpol } \\ \text { Nordpol }\end{array}\right\}$ der Spirale zunächst lag. Die Schwingungsweite wird näılich immer gröfser in der ersten, dritten und fünften der sechs Versuchsabtheilungen, in welchen der Wismuthstab $\left\{\begin{array}{l}\text { gesenkt } \\ \text { gehoben }\end{array}\right\}$ wurde bei der Bewegung des Magneten zu $\left\{\begin{array}{l}\text { höheren } \\ \text { niederen }\end{array}\right\}$ Scalentheilen; dagegen nehmen die Schwingungsweiten stetig ab innerhalb der zweiten, vierten und sechsten Versuchsabtheilung, in denen die ungekehrte Bewegung statthat.

Wurde dagegen ein dünner Eisendraht statt des Wismuthstabes inverhalb der Spirale auf - und abbewegt, so brachte er stets dic entgegengesetzte Ablenkung des Magneten bervor und zwar wurde letzterer nach $\left\{\begin{array}{l}\text { hölieren } \\ \text { niederen }\end{array}\right\}$ Scalentheilen getrieben, wenn der Eisendraht sich in der $\left\{\begin{array}{l}\text { oberen } \\ \text { unteren }\end{array}\right\}$ Lage befand.

8. Durch diese Versuche werden also die Beobachtungen Weber's vollkommen bestätigt. Dagegen aber bin ich aufser Stande einzusehen, wie das Resultat aus den Versuchen der HH. Hankel und Leyser möglich wurde, welches in Poggendorff's Annalen Bd: 67, S. 170 bis 172 mitgetheilt ist. Nach denselben soll sich chemisch-reines Wismuth, welches nicht von einem galvanischen Strone umflossen wird, zwischen den Polen cines borizontal - schwebenden Hufeisenmagneten verhalten wie ein Magnet, so zwar, dafs das dem Boden zugewandte Ende cinen bestimuten Pol des beweglichen Hufeisenmagneten anzieht, las abgewandte Ende denselben Pol zurückstöfst; die Dif- 
ferenz der Maguetstände in beiden Fällen beträgt 5 bis 7,5 der dortigen Scalentheile. Ist aber der Wismuthstab, wie in unserem Falle durch eine galvanische Spirale erregt, daun soll derselbe seine Polarität, nicht blofs während der Bewegung innerhalb der Spirale, sondern auch wöhrend er gehoben oder gesenkt ist, unkehren, und jetzt eine Differenz der Magnetstäude von 8,9 bis 9,4 Scalentheilen im ungekebrten Sinne zeigeu. Es ist zu bedauern, dafs die Stromesrichtung in der Spirale, oder die Art der Polarität des Wisnuthstabes oder des zur Probe benutzten Eisenstabes, oder die Lage der höheren und niederen Scalentheile gegen den Beobachter nicht angegeben ist: der Umstand, dafs " das angewandte Wismuthstück ohne Strom wie ein Eisendralit wirkt ", vom Strom umflossen aber die entgegengesetzte Wirkung hervorbringt, kann über die Richtung der Polarităten in beiden Fällen keineu Aufschlufs geben. Verhielt sieh vielleicht der Wismuthstab wie eiu permanenter Magnet? Ohne Stroun wurde er nur in einer Lage geprift, nicht aber nachdem sein oberes Ende nach uwten gekehrt worden. Oder hatte der Erdinagnetismus auf das Wismuth einen Einflufs wie auf das weiche Eisen geäufsert? Die beiden einzigen füglich erlaubten Conjecturen tiber dieses jedenfalls höchst merkwürdige Resultat sind durch die Bemerkungen abgeschnitten: "Das Wiswuth war chemisch rein, und so aufgehängt, dofs es an einem Faden auf- und abgezogen werden konnte, ohne dafs der Magnet im geringsten erschüttert wurd ".

Ich habe mich benüht init meinem Wismuthstabe eine Ableukung des Magneten hervorzubringen, wenn sich derselbe in der untbätigen und ungeschlossenen Spirale aufund abwärts bewegte, doch blieben alle Versuche olne allen Erfolg.

9. Waren nun auch meiue Versuche vollkommen übereinstinmend mit denen des Hru. Weber, so ist es eine andere Frage, ob diese Versuche berechtigt seyen, einen Nachweis über das Weseu dès Diamagnetismus zu führen. Es siud bcsonders zwei Bedeuken gegen diese Berechtigung, 
welche mich veranlafsten, eine beträchtliche Anzahl verschiedener Versuchsreihen auszuführen.

Erstens weist $\mathrm{Hr}$. W e ber an mehren Orten der citirten Abhandlungen darauf hin, dafs an verschiedenen Stellen im Innern einer cylindrischen galvanischen Spirale nur "nahe gleiche elektromagnetische Scheidekräfte wirken " ') und daf's sonach ein Wismuthstab in einer solchen Spirale "innerhalb gewisser Gränzen verschoben werden kann, ohne dafs diese Kräfte sich merklich ändern ". "Beträgt " nämlich " der Durchmesser z. B. den 40sten Theil der Länge, so ist in mehr als $\frac{7}{5}$ des ganzen von der Spirale umschlossenen Raumes die elektromagnetische Scheidungskraft bis auf 1 Procent constant, und in fast $\frac{2}{3}$ dieses Raumes ist sie bis auf $\frac{1}{r}$ Procent constant “. Dem hier angegebenen Verhältnisse ist aber weder bei den Versuchen des Hrn. Webe $r$ noch bei den von mir ausgeführten nacbgekommen, denn bei ersteren verhält sich der Durchmesser der Spirale zu deren Länge wie $1: 11,2$, bei letzteren wie $1: 12,7$. Und demgemäfs müssen die in denselben obwaltenden Scheidungskräfte noch weit gröfseren Aenderungen unterworfen seyn, als den angeführten. Ist aber dieses der Fall, dann kaun bei der Bewegung eines Leiters für galvanische Ströme innerhalb einer solchen Spirale unmöglich die Volta-Induction ${ }^{2}$ ) "ganz beseitigt" worden seyn.

Zweitens aber kommt es, wenn man über die Richtung der Polarität der kleinsten Theilchen das Wismuth entscheiden will, nicht darauf an, dafs das Wismuth durch eine galvanische Spirale erregt werde, sondern es kommt darauf an, da/s die erregende Kraft stärker auf die Mitte der Längsausdehnung eines wismuthmagnetischen Körpers wirke, als auf dessen Ende. Dieser Bedingung ist aber in den Versuchen des $H r n$. Weber nicht genügt. Der Wismuthstab bewegt sich innerhalb ciner Spirale von doppelter Länge auf und $a b$, und seine Polarität wird an den

1) Maafsbestimmungen S. 493 u. 548. Poggend orff's Annalen Bd. 87, S. 153.

2) Maafsbestimnungen $S .535$ u. 511 . 
resp. Enden seiner Bahn geprüf. Ist nun z. B. der Stab in dic untere Lage gebracht, so befindet sich sein obcres Ende in der Mitte der erregenden Spirale. In der Mitte hat aber die erregende Kraft derselben einen Maximumwerth '), folglich wird der Stab von seinem oberen Eude her särker erregt, als von seiner Mitte. Ebenso wird der Stab von seinem unteren Ende her stärker erregt, als von der Mitte, wenn er in die obere Lage gebracht ist.

10. Zur Prüfung des ersten der hier angeführten $\mathrm{Mr}$ gumente stellte ich folgende Ueberlegungen on. Un den Versuch von der Wirkung der Indactionsströme auf den beweglichen Magneten zu befreien, steben namentlich zwei Wege offen: entweder es werden die Inductionsströme so geregelt, dals sie gegenseitig ihre Wirkung auf den Magneten vernichten, und dieses kann im vorliegenden Falle dadurch geschehen, dafs man statt eines massiven Wismuthcylinders ein Bündel von sebr dünven mit Seide umsponnenen Wisunthcylindern in der Spirale auf-und abbewegt. Oder die Inductionsströıne werden gauz und gar vermieden, was geschieht, wenn man statt eines diamagnetischen Leiters einen dianaguetischen Isolator für galvanische Ströme dem Versuch unterwirft. Die erste dieser Proben unufste ich einstweilen aufgeben, weil es mir trotz aller Bemübungen unmöglich wurde, aus chemisch-reinem Wismuth binreichend dünne Cylinder von der Länge des benutzten stärkeren zu giefsen. Und diese Länge mufsten sie wenigstens anıähernd haben, wcun die zu erwartenden Resultate mit den durch den massiven Cylinder gewonuenen verglichen werdeu sollten. Es blieb also nur übrig, mich auf dic zwcite Probe zu beschränkell.

11. Versuch 9. Ein dem hiesigen physikalischen Cabinct gehöriges regelmälsig dreiseitiges Prisma von sehr reinem Faraday'schem schwerem Glase aus der Werkstatt

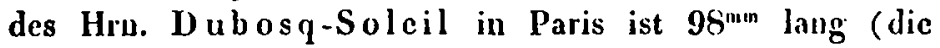
Wismuthcylinder, mit welchen $\mathrm{Hr}$. Weber die ursprünglichen Versuche ausführte, sind $92^{\text {inm }}$ lang ) und hält $28^{\text {nmm }}$

1) Maafobestimanungen S. 547. 
in Scite. Dasselbe wurde mittelst Seidenfäden iunerhalb der thätigen galvanischen Spirale analog dem Wiswuthcylinder des ersten Versuches bewegt. Wie aber auch diese Bewegungen abgeändert werden mochten, es war nicht möglich, weder die Schwingungen des Magueten zu mindern, noch den rubenden Magneten in Schwingungen zu versetzen. Der Strom in der Spirale war so stark, dafs ein Stückchen dünnster eiserner Klaviersaitendraht von der Lünge des Glasprismas den Magneten beim Heben zu deu böcbsten Scalentheilen (1600) und beim Senken zu deu niedersten (337) trieb. Ein Wismuthstab von gleicher Länge und nur $5^{\text {mm }}$ Durchmesser brachte durch abwechselndes Heben und Senken eine entgegengesetzte wiewohl sehr geringe Ablenkung hervor, als der Eisendraht. Durch 31 Hebungen und ebenso viele Senkungen war es möglich eine Schwingungsweite des Magneten von 15 Scalentheilen auf 0 herabzudrücken. Bei derselben Stromstärke verwochte der Wisuuthcylinder des ersten Versuches mittelst 10 Bewegungen cinen Ausschlag von 20 Scalentheilen zur Ruhelage herabzubringen.

12. Versuch 10. Statt des Wismuthcylinders vom erstew Versuch wurde ein Cylinder von reinem weifsem Waclıs in der Spirale bewegt und mit ilın die oben für den Wismuthcylinder durchgefübrte Versuchsreihe wiederholt. Eine Probe dieses Wachses verhielt sich zwischen den Polen eines Elektromagneten vollkommen diamagnetisch. Der Wachscylinder war $147^{\text {mun }}$, also fast genau so lang als der Wisıuthcylinder, er hatte aber $29^{\mathrm{mm}}$ Durchmesser und wog $155^{\mathrm{gr}}, 3$. Jedesmal, wenn eine Versuchsreihe für den Wismuthcylinder durchgeführt war, wurde der Wacliscylinder eingehangen, und mit ihm eiu analoger Versuch angestellt. Alle diese Reihen sagen dasselbe aus. Es ist oben No. 7 die der ersten Versuchsreilie entsprechende mitgetheilt unter der Ueberschrift $\approx 6$. Versuch mit dem Wachscylinder ". Ueber die Anordnung dieser Tabelle und die Anordnung des Versuches gilt dasselbe, was No. 7 über die Tabelle " $a$. Versuche wit dem Wismuthcyliuder" 
gesagt wurde. Von jenem regelmä/sigen Wachsen und $A b$. nehmen der Schwingungsbögen unter Einflufs des Wismuthcylinders ist nichts wieder vorzufinden in der Versuchsreihe mit dem Wachscylinder.

13. Diese Versuche mögen einstweilen gentigen, um zu zeigen, dafs diamagnetische Substanzen, welche in einer galvanischen Spirale auf - und abbewegt werden, keine Ablenkung eines benachbarten Magneten hervorbringen; wenn sie nicht zugleich Leiter für galvanischc Ströme sind, und wenu somit die Strome der Volta-Induction nicht zu Stande kommen können. Beruhte aber das Wesen des Diamaguetismus in inducirten Molecularströmen, so müfste auch beim Wachs die analoge Erscheinung eintreten, die wir beim Wismuth beobachteten. Diese Versuche lassen sich leicht erweitern, wenn man Schwefel, trockenes Holz, Schellack und andere Isolatoren den angegebenen Bedingungen unterwerfen wollte. Leider nöthigten mich Gründe von rein örtlicher Natur die Untersuchung zu beschleunigen.

14. Finde ich mich nun genöthigt, die in No. 7 beobachtete anorwale Polarität des Wismuth als eine scheinbare anzusehen, und die Ursache der Erscheinung in gewöhnlichen Inductionsströmen suchen zu müssen, so frage ich weiter: wie wird sich der bewegliche Hufeisenmagnet verhalten, wenn ihm die wismuthmagnetische Substanz unter Einflufs des galvanischen Stromes nicht wäbrend eines beständigen Auf- und Abbewegens, sondern im Zustand der Ruhe dargeboten wird? Zur Beantwortung dieser Frage stellte ich eine Anzahl von Versuchsreihen in folgender Weise an.

15. Versuch 11. Die im achten Versuch No. 7 angeführte Beobachtungsinethode wurde dahin abgeändert, dafs nicht, wie es dort geschah, der Wismuthstab bei jeder Schwankung des Magneten gehoben oder gesenkt wurde, sondern dafs derselbe während 20 einfacher Sch wingungeu des Magneten in der oberen Lage, während der nächsten 20 Schwingungen in der unteren, dann wieder ebenso lange in der oberen Lage u. s. f, verblieb. Die Beobachtungen 
wurden ohne Pause für die gauze Reihe fortgesetzt. Für jede Schwingung wurden die äufsersten Werthe aufgezeichnet, und das Mittel und die Differenz von je zwei nacbeiuander beobachteten Zahlen berechnet, sowie das Mittel aus jeden 20 fur die obere oder untere Lage erbaltenen Zahlen. Die berechneten Mittel je zweier unmittelbar nacheinander beobachteten Zahlen sind der Ausdruck für die Gleichgewichtslage des Magneten währẹnd der entsprechenden Oscillation; die berechneten Differenzen dagegen bezeichnen die zugehörigen Schwingungsweiten. Diese beiden Zahlenreihen sagten aber weiter nichts aus, als was schon der erste Versuch lehrt. In Moment, wo der Wismuthstab $\left\{\begin{array}{l}\text { gehoben } \\ \text { gesenkt }\end{array}\right\}$ wurde, rlickt nämlich die Gleichgewichtslage des Magneten auf $\left\{\begin{array}{l}\text { niedere } \\ \text { hölere }\end{array}\right\}$ Scalentheile; da aber dieser Wechsel in der Länge des Stabes nur vorgenommen wurde, wenn der Magnet vou böheren zu niederen Scalentheilen schwang, so wurde demgemäfs beim $\left\{\begin{array}{l}\text { Heben } \\ \text { Senken }\end{array}\right\}$ die Schwingungsweite plötzlich $\left\{\begin{array}{l}\text { vergrölscrt } \\ \text { verkleinert }\end{array}\right\}$. Diese Erscheinungen traten aber uur ein in Moment des Hebens und Senkens, und würden entgegengesetzt gerichtet gewesen seyn, weun statt des Wismuths ein Eisenstab substituirt worden wäre. Dagegen war weder in den Zahlen für Gleichgewichtslage und Schwingungsweite während des ruhenden Wismuthstabes, noch in der graphischen Darstellung derselben irgend eine besondere Regelmälsigkeit zu entdecken. Ich halte es daber für tuberllüssig, dieselben mitzutheileu.

Wichtiger dagegen sind diejenigen Mittelzahleu, welche aus jeden zwanzig Beobachtungen für die ganze Zcit der oberen respective unteren Lage des Wiswuthstabes berechnet wurden. In der folgenden Tabelle mögen diese Zahlen aus der ausgedehntesten der überhaupt ausgeftihrten Versuchsreilen zusammengestellt werden. In der ersten Columne ist die Lage des Wismuthstabes nit deu Worten "gehoben, gesenkt " bezeichnet, und die zweite Columne 
enthält die mittlere Gleiclgewichtslage des Magneten während dieser Stellungen des Wismuthstabes, berechnet aus je 20 beobachteten Ausschlägen des Magneten.

\begin{tabular}{|c|c|c|c|c|}
\hline \multirow{4}{*}{$\begin{array}{c}\text { Der } \\
\text { Cylinder } \\
\text { war }\end{array}$} & \multicolumn{4}{|c|}{ Vorsuche } \\
\hline & \multicolumn{2}{|c|}{ mil dem Vismutheylinder. } & \multicolumn{2}{|c|}{ mit dern Vachscylinder. } \\
\hline & \multicolumn{2}{|c|}{ Stand des Magneten. } & \multicolumn{2}{|c|}{ Stand des Magneten. } \\
\hline & $\begin{array}{c}\text { Mittel } \\
\text { aus } 20 \text { Beob- } \\
\text { achtungen. }\end{array}$ & \begin{tabular}{|c} 
Mittel \\
aus den letzten \\
10 Beobach- \\
tungen.
\end{tabular} & $\begin{array}{c}\text { Mittel } \\
\text { aus } 20 \text { Beob- } \\
\text { achlungen. }\end{array}$ & $\begin{array}{c}\text { Mitiel } \\
\text { nus den letzien } \\
10 \text { Beobach- } \\
\text { tungen. }\end{array}$ \\
\hline $\begin{array}{c}\text { gehoben } \\
\text { gesenkt } \\
\text { gehoben } \\
\text { gesenkt } \\
\text { gehoben } \\
\text { gesenkt } \\
\text { gehoben } \\
\text { gesenkt } \\
\text { gehoben } \\
\text { gesenkt } \\
\text { gehobrn } \\
\text { gesenkt }\end{array}$ & $\begin{array}{c}\mathbf{5 0 7 , 1 4 0} \\
\mathbf{5 0 7 , 5 6 5} \\
\mathbf{5 0 5 , 5 4 5} \\
\mathbf{5 0 6 , 0 3 0} \\
\mathbf{5 0 5 , 0 1 5} \\
\mathbf{5 0 5 , 1 1 5} \\
\mathbf{5 0 4 , 7 0 5} \\
\mathbf{5 0 4 , 7 2 0} \\
\mathbf{5 0 4 , 1 9 5} \\
\mathbf{5 0 5 , 6 8 5} \\
\mathbf{5 0 4 , 5 1 0} \\
\mathbf{5 0 4 , 5 8 5}\end{array}$ & $\begin{array}{c}507,25 \\
508,06 \\
505,54 \\
505,37 \bullet \\
505,11 \\
505,84 \\
504,57 \\
504,19 * \\
504,17 \\
505,13 \\
\mathbf{5 0 4 , 7 3} \\
\mathbf{5 0 4 , 5 5}\end{array}$ & $\begin{array}{c}496,372 \\
495,800^{*} \\
496,240^{*} \\
496,175 * \\
495,415 \\
495,810 \\
495,530 \\
494,675 * \\
494,230 \\
494,750 \\
494,395 \\
494,030^{*}\end{array}$ & $\begin{array}{c}496,38 \\
496,07^{*} \\
495,92 \\
495,37^{*} \\
494,93 \\
495,11 \\
495,60^{*} \\
495,21^{*} \\
494,94 \\
494,82^{*} \\
494,68 \\
493,53\end{array}$ \\
\hline
\end{tabular}

Wïrden wir keine weiteren Folgerungen aus den beobachteten Zahleu machen, als die in der zweiten Columne dargestellten, so würden wir allerdings zu dem Schlufs berechtigt seyn, dafs ein Wismuthstab, auch während er sich innerlalb einer galvauischen Spirale von mehr als doppelter Länge in Ruhe befindet, die entgegengesetzte Polarität eines Eisenstabes annimmt. Deun denken wir uns rom Fernrohr nach der Spirale sehend, so ist der Nordpol derselben oben, der Nordpol des Magneten links und die höheren Scalentheile ebenfalls links. Ein in der Spirale $\left\{\begin{array}{l}\text { gelubener } \\ \text { gesenkter }\end{array}\right\}$ Eisenstab würde demgemäls dem Magweten zunächst einen $\left\{\begin{array}{l}\text { Südpol } \\ \text { Nordpol }\end{array}\right\}$ haben, sonach den $\left\{\begin{array}{l}\text { Nordpol } \\ \text { Südpol }\end{array}\right\}$ des Magueten anziehen und somit würde der mit ihm verbundene Spiegel das Bild von $\left\{\begin{array}{l}\text { höheren } \\ \text { niederen }\end{array}\right\}$ Scalentheilen dem Fernrohre zuführen. Hier finden wir aber das Gegentheil: 
$\operatorname{dem}\left\{\begin{array}{l}\text { geliobenen } \\ \text { gesenkıen }\end{array}\right\}$ Wismuthstab entsprechen $\left\{\begin{array}{l}\text { niedere } \\ \text { lühhere }\end{array}\right\}$ Scalentheile. Doch kam ich auf die Vermuthung, es möchte, wenn auch nur ein grofser Theil dieser Differenzen, auf diejenigen Störungen zu schicben seyn, welche durch die Inductionsströıne beim Act des Hebens oder Senkens im inittleren Stande des Magneten bervorgebracht werden. Zu dem Ende berechnete ich die in der dritten Columne verzeichneten Mittel aus blofs den letzten 10 Beobachtungen während der resp. Lagen des Wismuths in der galvanischen Spirale. Es zeigte sich, dafs die mit einem (*) versehenen 3 Zahlen sich nicht mehr jener Regelmäfsigkeit im Steigen und Fallen unterwerfen, und es wird mindestens höchst zweifelhaft, ob sich unter den angegebenen Bedingungen im Wismuth eine Polarität mit Sicherheit auch dann noch nachweisen lasse, wenn es innerhalb einer solchen galvanischen Spirale nicht in Bewegung, sondern wenn es in Ruhe ist. Wenigstens würde es ungleich umfangreicherer und kräftigerer Apparate bedürfen, um einen solchen Nachweis zu führen.

16. Versuch 12. War eine der soeben beschriebenen Versuchsreihen beendet, so wurde der Wismuthcylinder mit dem schon früher gebrauchten Wachscylinder vertauscht und nun die Versuchsreihe unter sonst denselben Bedingungen wiederbolt. In der vorigen Tabelle ist unter der Ueberschrift "Versuche mit dem Wachscylinder" die der soeben beschriebenen Versuchsreihe entsprechende in ihren Resultaten angefügt. Auch bier sind die ursprünglich beobachteten Zahlen, sowie die darauf berechneten mittleren Magnetstände und Weiten der einzelnen Scbwingungen unterdrückt. Was dieselben betrifft, so war, wie schon der dritte Versuch aussagt, eine regelmäfsige Abnahme oder Zunalime im Moment des Hebens und Senkens nicht zu bemerken. Dagegren sind in der vierten Columne der vorigen Tabelle die Mittel ans je 20, sowie in der fünften die Mittel aus blofs den letzten 10 Beobachtungen angegeben Die Ausnahmen vou der mehrfach erörterten, "dia- 
magnetischen " Regel sind ebenfalls mit einem (*) bezeichnet. Und hatte auch der Wachscylinder nur den fünften Theil der Masse vom Wismuthcylinder, so dürften doch diese Ausnahmen in sonst so gut miteinander stimmenden Zahlen sich nicht derart häufen, als es hier geschehen ist. Diese Ausnahmen unterstützen den Ausspruch der vorigen Nummer, sie machen die Regel selbst zweifelhaft.

17. Aber gesetzt auch, ein Cylinder aus wismuthmagnetischer Substanz erhalte die entgegengesetzte Polarität eines Cylinders aus eisenmagnetischer Substanz, wenn er sich in der unteren oder oberen Hälfte einer galvanischen Spirale von mehr als doppelter Länge in Rube befindet: so ist damit noch nicht nachgewiesen, dafs er diese umgekehrte Polarität erhalte, wenn er in der Mitte seiner Längsausdehnung stärker erregt wird als an den Enden. Und das war es ja gerade, worauf es mir in den früher aufgestellten Ansichten über diamagnetische Wirkungsweise ankam. Ich hatte (Pogge ndorff's Annalen Bd. 82, S. $90 \mathrm{ff}$.) einen Versuch angeführt, der meine Ansichten bestätigte. Die Gültigkeit desselben wurde aber von Hrn. Weber (Mafsbestimmungen 536) in Zweifel gezogen. Die gegen dieselbe angeführten Gründe haben mich so sehr überzeugt, dafs ich es nicht mehr der Mühe werth hielt, meinen eigenen Versuch zu wiederholen. Dagegen aber benutzte ich die klassische Beobachtungsmethode Weber's, um die bisher durchgeführten Versuche so weit abzuändern bis sie meinen Zwecken genügten.

18. Versuch 13. Ein hohler Cylinder, aus 2 Lagen Pappe bestehend, wurde mit drei Lagen Flanell und hierauf noch mit mehreren Lagen Papier überklebt. Derselbe $N S$ - Taf. III, Fig. 4 - hatte bei $24^{\mathrm{mm}}, 5$ innerem Durchmesser einen äufseren Durchmesser von $41^{\mathrm{mm}}$ erhalten und war $440^{\mathrm{mm}}$ hoch. Ueber den mittleren Theil desselben wurden $1 \mathrm{kgr}, 87$ mit Seide übersponnener und gefirnifster Kupferdraht gewunden. Der Draht war in 11 Lagen vertheilt und diese enthielten, um die magnetische Scheidungs- 
kraft nröglichst in der Mittc zusammenzudrängen, bezüglich $117+105+101+95+89+82+74+66+57+44$ $+33=863$ Windungen. Die unterste Lage batte cine Höhe von $190^{\text {nom }}$ und jede Lage war von der nächsten durch zwei Schichten gefirnifster Seide getremut. Innerhalb der Papprolle hing der schon mebrfach gebrauchte Wismuthcylinder an einem Seidenfaden von der Decke des Zimmers herab, und konnte ohne anzustofsen in beliebig grofsen Intervallen aus der Entfernung auf - und abgezogen werden. Vor dieser Spirale hing, ebenfalls von der Decke des Zimmers herab, eine Latte an einem Bündel ungedrehter Scidenfäden, welche aufser dem Spiegel $\boldsymbol{P}$ ein astatisches System von zwei Magnetstäben (212 wum lang und $26^{\text {mm }}$ in Seite) $N_{1} S_{1}$ und $S_{2} N_{2}$ trug. Und zwar war, wie es die Figur andentet, der obere Magnetstab $N, S$, auf der rechten Seite, der untere auf der linken Seite der Latte angeschraubt, so dafs ihre Pole $S_{1}$ und $N_{2}$ gerade über und unter den Polen der Spirale, jedoch auf verschiedenen Seiten derselben schwebten, und noch Raum genug zu horizontalen Oscillationen des astatischen Systemes übrig blieb. Die Spiegelfläche $P$ warf in bekannter Weise das Bild einer $2^{\text {nutr,j, }}$ entfernten in Millimeter getheilten Scale in ein iiber derselben stehendes Fernrohr. Jic höheren Scalentheile befanden sich zur Linken des durch das Fernrohr sehenden Beobachters. Wie in der vierten Versuchsreihe wurde auch hier der Wismuthstab imnerhalb der Spirale so weit gehoben, bis sein unteres Ende genugsam ans dem Bereich des Poles $\mathrm{N}_{2}$ entfernt war, und für 20 einfache Schwingungen abwechselnd der niederste und höchste Stand im Fernrolir beobachtet. Dasselbe geschah für die folgenden 20 Schwingungen, naclsdem der Stab gesenkt worden war, bis sein oheres Ende aus dem Bereich des Poles $S$, kam u. s. f. Aus jeden 20 nacheinander bcohachteten Zahlen wurde das Mittel genommen, und demnächst wurde das Mittel berechnet aus den für die obere Lage des Wisınuthstabes crhaltenen Mittelzahlen, sowie das Mittel aus den Zahlen für die untere Lage. In der folgenden Tabelle mögen 
mögen die so gefundenen Werthe von 4 Versuchsreihen aufgeführt werden.

\begin{tabular}{c|c|c|c}
$\begin{array}{c}\text { Anzahl der He- } \\
\text { bungen des Wis- } \\
\text { muth. }\end{array}$ & $\begin{array}{c}\text { Mittel aus allen in } \\
\text { der obern Lage } \\
\text { beobachteten } \\
\text { Zahlen. }\end{array}$ & $\begin{array}{c}\text { Anzalil der Sen- } \\
\text { kungen des VVis- } \\
\text { muth. }\end{array}$ & $\begin{array}{c}\text { Mittel aus allen in } \\
\text { der untern I,age } \\
\text { beobacliteten } \\
\text { Zahlen. }\end{array}$ \\
\hline 9 & 555,300 & 8 & 554,590 \\
9 & 492,997 & 8 & 492,244 \\
10 & 502,115 & 9 & 502,011 \\
12 & 547,740 & 11 & 548,410
\end{tabular}

Drei dieser vier Reihen zeigen, dafs die Zahlen der mittleren Stände des Magnetsystemes für den gehobenen Wismuthstab gröfser sind, als für den gesenkten Stab. Und so mufste es der Fall seyu, wenn das Wismuth innerbalb der galvanischen Spirale eine gleichgerichtete Polarität annimwt wie das Eisen. Die letzte Reihe jedoch zeigt eine gröfsere Zahl für den gesenkten Stab als für den gehobenen, und da ich den Grund dieser Abweichuvg nicht zu ermitteln vermag, scheue ich mich nicht, sie den übrigen Reihen gegenüber zu stellen. Ueberdiefs siud die Zahlenunterschiede zu klein, als dafs ich mich hätte mit diesen Versuchen begnügen sollen. Die Nachtheile des Apparates mufsten mir bald einleuchten. Sie bestanden namentlich darin, dafs der Wismuthstab nicht weit genug aus dem Bereich des einen Magneten entfernt werden konnte, wenn er auf den andern wirken und dabei immer an seinen Enden schwächer erregł werden sollte, als zwischen denselben. Ich stellte deshalb nur wenige Versuchsreihen mit diesen Apparat an und nahm aus den Beobachtungen eiuzig die Versicherung, da/s eine normale Polarität des Wismuths nachweisbar seyn könnte.

19. Versuch 14. Der Spiegel, die Bewegung des Wismuthcylinders und das astatische Maguetsystem hatten sich bewährt, wenngleich es vortheilhaft erschien, die schweren Magnetstäbe mit leichteren zu vertauschen. Dagegen aber wurde es nothwendig, statt einer einzigen galvanischen Spirale deren zwei von entgegengesetzten Seiten her auf Poggendorff's Annal. Bd. XCII. 
das astatisclue System wirken zu lassen, so zwar, dafs beide allein sich gegenseitig compensiren, dafs aber das Magnetsystem zu Gunsten (oder zum Nachtheil) der cinen von beiden ausschlägt, wenn dieselbe mit Wismuth gefüllt wird. Demgemä[s stellte ich auf einen Holzkasten $Q R$ ('Taf.III Fig.5) zwei galvanische Spiralen $N S$ und $N_{1} S_{1}$ auf, so dafs ihre Axen vertical standen und etwa $218^{\mathrm{mm}}$ von einander entfernt waren. Diese Spiralen waren auf hohle Holzcylinder von geringer Wanddicke aufgewunden und wurden durch quadratische Holzplatten zusarnmengehalten, die eine Axenlänge von $108^{\mathrm{mm}}$ zuliefsen. Die Spiralen waren aus gefirnifstem und mit Seide umsponnenem Kupferdraht dargestellt, hatten einen inneren Durchmesser $=42^{\mathrm{mm}}$ und einen äufsereu $=92^{\mathrm{min}}$ und bestanden jede aus $597 \frac{1}{2}$ Windungen, welche in je 11 mit gefirnifster Seide getrennten Lagen vertheilt wareu. In der Spirale $N S$ sollte der schon mehrfach benutzte Wisınuthstab $W$ magnetisirt und entmagnetisirt und zu dem Eude gehoben und gesenkt werden. Un die Wärmeleitung möglichst zu mindern, wurde sie mit einem Hohlcylinder $\boldsymbol{A} B$ von zwei Lagen Pappe und drei Lagen Flanell ausgefüttert, der gerade in den hohlen Holzcylinder der Spirale pafste, und den inneren Raum bis auf $24^{\mathrm{mm}}, 5$ Durchmesser verengte. Die Länge dieses Cylinders betrug $440^{\mathrm{mm}}$ und er ragte über der Spirale so weit hervor, dafs er den Wismuthcylinder in dessen oberer Lage noch gerade überdeckte; unter der Spirale war er durch eine Oeffnung im Deckel $Q R$ des Kastens gesteckt, sicherte so die Stellung der Spirale $N S$, diente als Führung für den Wismuthstab und schützte denselben wenigstens etwas gegen $\mathbf{A b}$ kühlung, wenn er in dem Bereich der Spiralc erwärnt und demnächst in die untere Lage gebracht worden war. Mitten zwischen beiden Spiralen schwebte das astatische Magnetsystem, bestehend aus zwci Stäben $N_{2} S_{2}$ und $N_{3} S_{3}$ von $100^{\mathrm{mm}}$ Länge und $6^{\mathrm{mm}}$ Durchmesser. Dieselben waren durch einen Streifen dicker Pappe gesteckt, und zwischen ihven war auf demselben Streifen der Spiegrel $P$ befestigt. Dicses astatische System hing an einem doppelten Coconfaden von 
cinem Dreifufs herab, während die Rolle, um welche sich die Schnur des Wismuthstabes bewegte, an die Decke des Zimmers geschraubt war. Die Polaritäten der Spiralen und der Magnete sind durch die Buchstaben $N$ und $S$ bezeichnet. Ein Blick auf die Figur lehrt, dafs, wenn die Spiralen in Thätigkeit versetzt werden, alle vier Pole derselben gleichmäfsig auf die Pole der Magnete derart wirken, dafs das bewegliche System sich von $P$ abwärts, senkrecht zur Verbindungrslinie beider Spiralen bewegt. Um diesen Uebelstand möglicbst zu beseitigen, wurde ein sehr schwerer Spiegel $P$ angewandt, er wog nämlich mit Einschlurs des übrigen blofs hemmenden Antheiles $243^{\mathrm{gr}}, 2$, während beide Magnete ein Gewicht $=47^{k^{r}}, 8$ hatten. So wurde es möglich durch kleine Verrückungen der Spirale $N_{1} S_{1}$ senkrecht zur Verbindungslinie beider Spiralen die Schwingungsdauer des astatischen Systemes willkührlich zu vergrölsern und zu verkleinern, und durch Verrückung in dieser Linie dem Spiegel $P$ eine willkührliche Richtung zu geben. Der Spiegelflüche $P$ gegenüber war in der Horizontalentfernung von $2^{\text {matr }}, 297$ die in Millimeter getheilte Scale und über derselben das Fernrohr aufgestellt. Die gröfseren Scalentheile befanden sich links von dem am Ferurohr stehenden Beobachter. Der Stroin von sechs Grove'schen Elementen unit je 80 Quadratcentm. Platinblech ging nacheinander durch beide Spiralen. Jie Constanz des Stromes wurde durch eine eingeschaltete Tangentenbussole erprobt. Bei einigen Versuchen überzeugte ich mich, dafs die mittlere Lage des Spiegels keine störende Aenderung erlitten hatte, wenn auch die Nadel der Tangentenbussole un melirere Zelintel-Grade zurückgewichen war. Sonach konnte auch eine geringe Aenderung in der Stromstärke, welche durch die Abkühlung der Spirale $N S$ in Folge des gehobenen Wismuthstabes veranlafst wurde, keinen Einflufs auf die Stellung des Magnetsystemes ausüben, noch dazu die Wärmeleitung durch die angegebenen Vorsichtsmifsregeln aufs äufserste vermindert worden war. Nachdem nun die durch Herstellung des Stromes entstandeven gröfseren Schwan- 
kungen des Spiegels in gecigneter Weise gedämplt worden waren, wurde der Wismuthstab in der Spirale $N S$ so hoch gehoben, dafs er oben und unten gleich weit aus derselben hervorragte und demnächst wurden dic äufsersten Scalentheile für 20 einfache Oscillationen abgelesen. Hicrauf wurde der Wismuthstab gesenkt, bis er sich weit aufserhalb der Spirale befand und abermals die Ablesung für 20 einfache Oscillationen bewirkt; hierauf wurde der Stab wieder gehoben, gesenkt u. s. f. Bei einigen so angestellten Versuchsreihen wurden die Magnete in der Weise benutzt, wie es die Figur angiebt, bei anderen wurden sie mit kupfernen Dämpfern ungeben. Gewannen auch im letzteren Falle die beobachteteu Zahlen an Gleichförmigkeit, so sagten die Resultate aus beiden Beobachtungsweisen und von allen Versuchsreihen einstimunig dasselbe aus, nämlich:

ein Wismuthstab nimmt zwar eine weit schwächere aber eine einem Eisenstab gleichgerichtete Polarität an, wenn er in der Mitte seiner Längsausdehnung eine stärkere magnetische Erregung erfährt, als an seinen Enden.

Zun Beweis des Gesagten mögen in der folgenden Tabelle die Resultate einer Beobachtungsreihe zusammengestellt werden. Der Stand der Tangentenbussole war $32^{\circ}, 5$.

\begin{tabular}{|c|c|c|c|c|}
\hline & \multicolumn{4}{|c|}{ Der Wismuthstab war } \\
\hline & \multicolumn{2}{|c|}{ gehoben. } & \multicolumn{2}{|c|}{ gesenkt. } \\
\hline & $\begin{array}{l}\text { Mittel aus } \\
20 \text { Beobach- } \\
\text { tungen. }\end{array}$ & $\begin{array}{c}\text { Mittel aus } \\
\text { den letzten } \\
10 \text { Beobach- } \\
\text { tungen. }\end{array}$ & $\begin{array}{l}\text { Mittel aus } \\
20 \text { Beobach- } \\
\text { tungen. }\end{array}$ & $\begin{array}{c}\text { Mittel aus } \\
\text { den letzten } \\
10 \text { Beobach- } \\
\text { tangen. }\end{array}$ \\
\hline $\begin{array}{l}1 . \\
2 . \\
3 . \\
4 . \\
5 .\end{array}$ & $\begin{array}{l}377,830 \\
\mathbf{3 7 2 , 4 2 5} \\
\mathbf{3 7 8 , 4 0 0} \\
\mathbf{3 6 5 , 1 0 5} \\
\mathbf{3 7 4 , 6 8 5}\end{array}$ & $\begin{array}{l}\mathbf{3 7 2 , 0 5} \\
\mathbf{3 8 2 , 0 8} \\
\mathbf{3 7 7 , 7 8} \\
\mathbf{3 6 2 , 8 0} \\
\mathbf{3 6 8 , 2 5}\end{array}$ & $\begin{array}{l}\mathbf{3 7 8 , 3 9 0} \\
\mathbf{3 8 2 , 9 3 0} \\
\mathbf{3 8 5 , 0 8 0} \\
\mathbf{3 8 1 , 1 7 5}\end{array}$ & $\begin{array}{l}379,26 \\
386,02 \\
386,07 \\
387,16\end{array}$ \\
\hline Mittel & 373,689 & 372,59 & 381,894 & 382,38 \\
\hline
\end{tabular}

Die erste Columne enthält die Zahl der vollführten Hebungen des Wismuthstabes, die zweite das Mittel aus 
den Beobachtungszahlen für je 20 einfache Oscillationen der Magnete, sowie die dritte das Mittel aus den letzten 10 beobachteten Zahlen für den gehobenen Stab. Ingleichen enthalten die vierte und fünfte Columne die entsprechenden Mittel für die zwischen je 2 Hebungen stattgehabte Senkung des Stabes. In der untersten Horizontalreihe sind endlich die Mittel aus allen in jeder zugehörigen Verticalreihe stehenden Zahlen verzeichnet. Der Vergleich der Zahlen in der zweiten und vierten oder der dritten und fünften Verticalreihe zeigt, dafs bei gehobenem Wismuthstab kleinere Zahlen beobachtet wurden, als bei gesenktem, ganz wie dieses bei einem eingebrachten Eisenstab der Fall war. Nur die zweite Zalıl in der dritten Reihe macht eive (zwar nur scheinbare) Ausnabme '). Der Grund davon war jedoch nicht zu ermittclu. Der Vergleich der Zahlen in der ersten mit denen in der zweiten Verticalreihe zeight, dafs (mit Ausnahme jener zweiten Zahl in der dritten Reihe) die während der aufwärtsgebenden Bewegung des Wismuthstabes stattgelıabten Inductionsströme eine Vergröfserumg. der Mittelzahlen hervorbrachten. Ebenso wurde eine Verkleinerung dieser Zahlen bewirkt durch die beim Senken des Stabes eutstandenen Ströme. Dasselbe tritt in den Mittelzahlen der untersten Horizontalreihe hervor. Dem entsprechend hat nun der Wismuthstab eive Verrückung

1) Haupıäachlich wegen dieser zwciten Zahl in der dritten Verticalreihe füge ich in Fig. 6 'Taf. Ill eine graphische Darstellung der Ergebnisse dieser Tabclle hinzu. In derselben stellen die Oddinaten der Linitu $A B, a b, C D$ und $c d$ die Zahlen resp. der zwciten, dritten, vierten und fünften Verticalreihe dar, wïlırend dic $A$ bscissen proportional den Zahlen der crsten Reihe sind. Durrla diesc Darstellung wird es augenscheinlich, dals die von dem Einflufs der Inductionsstülsc befreite linie $a b$ und $e d$ im Allgenueinen aufserhalb den Linien $A B$ und $C D$ liegen, wulche die Wirkung der Inductionsstöfse nuch mit enthahten. Forner zeigt sich, dafs tratz der genannten Zahl, doch dic Linie $c d$ noch gänzlich über und aufserlılb der Linie $a b$, wie $C D$ über $A B$ liegt. Fndlich wird der Einflufs des Wismuthstabes auch noch dadurch markirl, daf's dic unter demsclben entstandenen Curven $A B$ und $a b$ eine viel unregelmaifsigere Gustalt haben, als die ohae denselben cntstandenco Curven $C D$ und $c d$. 
des Magnetsystemes bewirkt von $381,894-373,689=8,205$ Scalentheilen, wenn man das Mittel aus allen beobachteten Zahlen als mafsgebend betrachten will.

Es ergiebt sich aber eine Drebung der Magnete von $352,38-372,59=$

$$
\text { 9,79 Scalentheilen, }
$$

wenn man die vou der Wirkung der Inductiousströme befreiten Mittel aus den jedesmaligen letzten Hälften der beobachteten Zahlen in Betracht zieht. Nach der Versuchsreihe wurde statt des Wismuthstabes cin weicber Eisendraht von gleicher Länge und $0^{\mathrm{gr}}, 365$ Gewicht in der Spirale gehoben und gesenkt. Die für die obere Lage beobachtete Mittelzahl betrug 192,5, die für die untere Lage beob. achtete Mittelzabl 309,0. Der Eisendraht brachte also eine Drehung von

\section{6,5 Scalentheilen}

hervor, welche Zahl ein ungefähres Maals für die Empfindlichkeit des Apparates, sowie in Vergleich mit 9,79 für die Menge des im Wismuth frei gewordenen Magnetismus abgeben mag. Ich spreche ron einew ungefähren Maafs, denn ich bin weit entfernt diesen Untersuchungen, ausgeführt mit den rolısten Hülfsunitteln, mehr als einen beobachteuden C.barakter beilegen zu wollen. Für messende Versuche scheue ich annoch Opfer und Mühe, da ich sie woch nicht für zeitgemäfs erachte. Als besonders schlagenden Beweis für meine Ansicht verdient aber nochmals hervorgehoben zu werden, da/s die con den Inductionsstö/sen befreiten Mittel aus den letzten 10 Einzelbeobachtungen eine Vergröfserung der normalen Polarität nachueisen, während die entsprechenden Mittel der 15. Nummer eine Verminderung der anormalen Polaritat desselben Stabes anzeigen.

20. Aufser jenen, mehr oder weniger indirecten Beweisen für die normale Polarität der kleinsten Theilchen in wismuthmaguetischen Körpern war mir noch ein directer Beweis äufserst wünschenswerth. Ich schlofs, wenn ein iu ciner galvanischen Spirale ruhender Wismuthstab cincn 
in seiver Nähe beweglich aufyehangenen Magueten abzulenken im Stande ist, so muls auch ungekelurt cin in einer galvanischen Spirale beweglich aufgehangener Wismuthstab vou eivem in seiner Nachbarschaft in Rube befindlicheu Magueten abgelenkt werden. Zur Bestätigung verfulr ich folgendermafsen: Die Spirale der 18. Nummer (Fig. 6) wurde (olne das wollene Futter) horizontal, und in dieselbe der vielfach benutzte Wismuthstab gelegt. Die bervorragenden Enden desselben wurden in Schlingen von fufslangen Seideuschuüreu gchangen, welche von eivem ebenfalls horizontalen dünuen Ilolzstab herabreichten. Dicser letztere war unit einem verticalen Spiegel in fester Verbindung und hing in seiner Mitte an einem Büudel roher Seidenfäden von der Decke des Zimmers herab. Durcb willkührliche Verkürzung der Schnüre konnte der Wismuthstab gehoben und frei schwebend in der Axe der Spirale erhalten werden. Rechts und links von der Spirale wurden Stahl - oder Elektromaguete, mit ihren entgegengesetzten Polen nach dem Wismuthstab scheud, aufgestellt, und dieser wurde durch einen starken galvanischen Strom magnetisirt. Die Ablenkung des Wismuthstabes und des mit ihm verbundenen $S$ piegels beobachtete ich durch Fernrohr und Scale. War nun der Wismuthstab in der unthätigen Spiralc zur Ruhe gekommen und wurde der Strom durch die letztere gesandt, so gerieth der Stab in Bewegung und belielt diese Bewegung nach rechts und links Stunden lang bei, ohue auch nur cine Tendenz zu irgend eince Ruhelage zu verrathen, mochte die Stromesrichtung und die lage, Zahl und Stärke der Magnete seyı, welche sie wollten. Wie ich mich später uiberzeugte, leiteten die anfänglichen Inductionsstöfse diese Bewegung cin, und die Ströıne der in der Spirale erwärmten luft unterhalten sie, während die Maguete nicht stark genug waren, dem schwe. ren Wismuthstab eine dauerude Richtung zu geben. Dennoch aber leitete mich dicses unerwartete Resultat irre, so dafs mir erst in Laufe der manuigfaltigsten Abäuderungen des Versuches klar wurde, wic ich ja wesentlich nichts an- 
deres hezweckte, als eine Darstellung des Versuches, welchen Hr. Poggendorff am 16. Dec. $1847^{\prime}$ ) der Akademie zu Berlin vorlegte, und welcher von Hru. T y udall wiederholt ${ }^{2}$ ) aber nicht ganz bestätigt gefunden wurde. Dieser Versuch besteht darin, dafs zwischen den Polen eines Elektromagueten $N$ und S. Taf. III. Fig. 7, eine galvanische Spirale $\nu \sigma$ mit ihrer Axe senkrecht zur Verbindungslinie der Pole aufgestellt, in dieselbe ein Stäbchen von Wismuth oder anderen Substanzen horizontal beweglich aufgehangen, und die Ablenkung beobachtet wurde, welche dasselbe durch die Spirale und die Magnetpole zugleich erfuhr. Unter denselben Umständen nun, unter denen Hr. Poggendorff eine Ablenkung nach der Richtung des Pfeiles $a$ beobachtete, fand Hr. Tyndall gerade die entgegengesetzte Ablenkung, näulich in der Richtung des Pfeiles $b$. Beide Forscher hatten den Magnetpolen die hauptsächlich erregende Wirkung zugewiesen, währeud der Spirale der untergeordnete Einflufs, nämlich die Ablenkung des schwebeuden Stäbchens, übrig blieb. Denngemäfs erklärte Hr. Poggendorff die beobachtete Richtung so, dafs die Magnetpole in den nächsten Stellen des aequatorial gestellten Wismuthstäbchens gleichnamige Pole erregten, und die Spirale wach den bekanuten Gesetzen eine Ablenkung des so entstandeneu Transversalmagneten bewirke. Hr. Ty udall dagegen wandte die F a rada y'sche Anschauung auf die von ihm beobachtete Ablenkung an, dafs nämlich die wismuthmagnetischen Substanzen sich vach den Stellen der geringsten, die eisenmagnetischen aber wach den Stellen der gröfsten Kraft im Magnetfelde bewegen. Nachdem es aber gelungen war, die normale Polarisirung des Wismuth durch die galvanische Spirale nachzuweisen, konute mich weder die eine noch die andere Erklärungswcise befriedigen, und desbalb unternalım ich eine neue Untersuchung des fraglichen Gegenstandes.

1) Poggendorff's Annalen Bd. 73, S. 475.

2) Philos. Mag. Ser. IV. Vol. It, p. 233 und Poggendorff's Annalen Bd. 87, S. 189. 
21. Versuch 15. Es wurden $210^{\text {mtr }}$ mit Seide übersponnenen und lackirten Kupferdrahtes von etwa $1^{\mathrm{mm}}$ Durchmesser und einschliefslich der Umspinnung von $1^{\mathrm{kgr} r}, 3 \mathrm{Ge}-$ wicht zu einer Spirale aufgewunden, die wesentlich aus 2 Theilen $\nu$ und $\sigma$ bestand und von oben gesehen etwa der Darstellung in Taf. III Fig. 8 glich. Die Spule, auf welche der Draht gewunden, war $83^{\mathrm{mm}}$ lang, hatte $40^{\mathrm{mm}}$ inneren Durchmesser und die geringste Entfernung der beiden Spiralhälften betrug $20^{\mathrm{mm}}$. Die Einrichtung derselben war gerade diese, damit zugleich die Pole eines sehr grofsen Elektromagneten $N$ und $S$ dem zwischen ihnen schwebenden Körper $w$ möglichst angenähert werden, und zugleich die Spirale denselben möglichst stark erregen konnte. Um die Luftströmungen abzuhalten, wurde die Vorrichtung so gut es ging mit einem Glasgehäuse umgeben. In der Spirale $\nu \sigma$ wurden nun an einem durch eine oben befindliche Oeffnung gezogenen möglichst dünnen Coconfaden folgende Substanzen in horizontaler Ebene beweglich aufgehangen:

Weifses Wachs - ein Cylinder $85^{\mathrm{mm}}$ lang, 22 ${ }^{\mathrm{mm}}$ Durchmesser und etwa $33^{g r}, 9$ schwer.

Schwefel - ein Cylinder 107 $7^{\mathrm{mm}}$ lang, $15^{\mathrm{mm}}$ Durchmesser, $38^{\mathrm{gr}}, 5$ schwer, aus Schwefelblumen gegossen

Knochen - ein Cylinder $88^{\mathrm{mm}}$ lang, $6^{\mathrm{mm}}$ Durchmesser, $5^{\mathrm{gr}, 3}$ schwer

Gewöhnliches reines Wismuth -1 ) ein Cylinder $39^{\mathrm{mm}}$ lang, $6^{\mathrm{mm}}$ Durchmesser, 10 ${ }^{\mathrm{gr}}, 3$ schwer - 2) ein Cylinder $100^{\mathrm{mm}}$ lang, $5^{\mathrm{mm}}$ Durchmesser, $20^{\mathrm{gr}}, 7$ schwer 3) ein Bündel aus 12 Stäben, ähnlich dem vorigen, von denen jeder einzeln mit Seide umsponnen und dann alle zusammengebunden waren, 4) ein Cylinder $51^{\mathrm{mm}}$ lang, $17^{\mathrm{mm}}$ Durchmesser und $111^{\mathrm{gr}^{\mathrm{r}}, 3}$ schwer 5) eine Platte $59^{\mathrm{mm}}$ lang, $31^{\mathrm{mm}}$ breit, $7^{\mathrm{mm}}$ dick und $122^{\mathrm{gr}^{\mathrm{r}}}, 5$ schwer, deren kürzeste Dimension dem Aufhängefaden parallel war.

Neusilberdraht ctwa $100^{\mathrm{mm}}$ lang und $1^{\mathrm{mm}}$ Durchmesser. Alle wurden nach der Richtung des Pfeiles a abgelenkt, 
wenn $N$ und $\nu$ die Nordpole und $S$ und $\sigma$ die Südpole des Magneten und der Spirale waren, wenn also in den oberen Drähten der Spirale die Strüme die Richtung der Pfeile $v$ und $\sigma$ hatten. Sie erfubren aber die entgegengesetzte Ablenkung; wenn entweder die Polarität des Magneten oder die der Spirale die entgegengesetzte wurde. Ich benutzte 22 Grove-Poggendorf'sche Elemente zur Erregung von Magnet und Spirale, so zwar, dafs ich an der Spirale ein zu einem Elemente säulevartig ordnete, an Magneten dagegen immer Doppelelemente benutzte. Zucrst wurde demnach die Spirale durch cine Säule von (1 oder) 2 einfachen Elementen, der Magnet durch eine Säule von 10 Doppelelementen erregt, und alle Substanzen geprüft; hierauf wurde vom Magneten ein Doppelelement genommen und durch dasselbe der Strom in der Spirale verstärkt, so dafs dieser jetzt durch 4 civfache Elemente erzeugt wurde, und abermals die ganze Reihe der Kör. per geprüft u. 8. f. Icl konnte bei dieser Gelegenheit beobachten, da/s die Körper immer stärker in der angegebenen Richtung a abgelenkt vourden, eine je stärkere Erregung die Spirale und eine je schwöchere der Magnet orfuhr. Das nagnetische Neusilber stellte sich allerdings vor Erregung der Spirale nicht in die aequatoriale Lage, sondern strebte, soweit es konnte, sich diagonal zur Hülse derselben gegen die Pole des Magncten zu lehuen. War aber z. B. sein vorderes Ende gegen den Nordpol $N$ des Magueten gewendet und wurde die Spirale durch einen Strom von der Richtung des Pfeiles $\nu$ erregt, so bewegte sich das Neusilber aus dieser seiner Lage und kain erst wieder zur hube, wenn sich sein vorderes Ende an den Südpol und das hintere an den Nordpol stützte. Es liegt nahe, ans diesen Versuchen zu schliefsen, da/s bei der angegebenen Einrichtung das grö/ste magnetische Moment nicht von den Magnetpolen, sondern vielmehr von der Spirale in den Körpern hervorgerufen wird. Nagg nämlich auch immerhin die absolute Wirkung des Magneten cine kräfti- 
gere seyn, als die der Spirale, so ist doch das Moment der durch den Magneten erregten Kraft ein geringeres, weil der zu prüfende Körper dem Einflufs des Magneten die kurze, dem Einflufs der Spirale hingegen die lange Abmessung darbietet. Wird aber die Einrichtung so getroffen, dafs magnetische Körper (wie schwarzer Schiefer oder ein Eisenstäbchen in der 11. Nummer der Ty ndall'schen Abbandlung) oder diamagnetische Körper, die sich mit der Längsausdehnung von Pol zu Pol stellen (wie ein senkrecht zur Hauptspaltungsrichtung geschnittenes Wismuthstäbchen in No. 6 und 8 derselben Abhandlung) sich ganz frei im Innern der Spirale bewegen können, und haben sich diese Körper zwischen den erregten Polen des Magneten eingestellt, dann wird die demnächst in Thätigkeit versetzte Spirale eine Ablenkung im Sinne des Pfeiles $b$ hervorbringen, wenn der Strom in ihr die durch die Pfeile $v$ und $\sigma$ angegebene Ricbtung einbält. Die Spirale bringt dann an dem magnetisirten Stäbchen dieselbe Ablenkung hervor, wie ein Galvanometerdraht an der Magnetnadel. Die nächstfolgenden Versuche haben mich von der Richtigkeit dieser Ansicht überzeugt, wemn ich auch leider nicht im Stande war, mir krystallisirtes Wismuth verschaffen zu können. Was aber die Angabe des Hrn. Tyndall ( $\$ 12$ der citirten Abhandlung) betrifft, in Folge deren gewöhuliches, sich in die Richtung der Spiralenaxe, also zu $N$ und $S$ aequatorial, einstellendes Wismuth ebenfalls die Ablenkung $b$ erfahren soll, so habe ich mich vergebens bemüht dieses Resultat zu gewinnen. Namentlich glaubte ich durch eine sehr schwache Kraft der Spirale gegenüber einem besonders starken Magneten, oder durch den oben bezeichneten kurzen und dicken Wismuthcylinder, sowie durch die breite Wismuthplatte die Ablenkung $b$ erzielen zu können, aber es ist mir in keiner Weise gelungen. Nur bisweilen entstand diese Ablenkung im Moment der Herstellung des Stromes in der Spirale, mufste aber den dabei hervortretenden Inductionsströmen 
zugewiesen werden, indem sic nur momentan auftrat, und sogleich in die entgegengesetzte Ablenkeng dauerud umschlug.

22. Mit dem in der 11. Nummer erwïhnten Flintglasprisma, sowie mit einem Stück Glasröhre ( $97^{\mathrm{mm}}$ lang und $11^{\text {mun }}$ Durchmesser) konnte ich keine Ablenkung erreichen. Wenn ich aber einen klcinen Cylinder ron chemisch reinem Zink oder cin Parallelepiped von Neusilber $\left(41^{\text {min }}\right.$ lang, $6^{\text {mm }}$

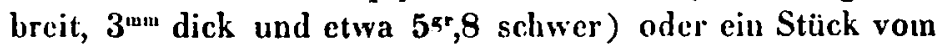
ordin̈̈rsten braunen Siegellack oder cin Stück starken Platinclraht zwischen den Maguetpolen in der Spirale aufhing, erhielt ich stets die Ableukung $b$, mochte das Verhältnifs der Erregung von Maguet und Spirale seyn, welches es nur immer wollte. Zwischen den Magnetpolen allcin stellten sich diese Substanzen alle axial. Um dieses ganz unerwartete Resultat zu ergründen, hing ich ein Stückchen weichen Eisendraht auf und fand, dafs es entweder in die eine oder in die andere Hälfte der Spirale gezogen wurde, sobald der Strom sich durch dieselbe bewegte. Daraus ging hervor, lafs der Ort, wo sich der Drelipunkt des aufgebavgenen Körpers befand, eine Stelle labilen Gleichgewichtes gegenüber der Spiralwirkung war. Es konnte demnach möglicherweise die cigenthümliche zweitheilige Form der Spirale den Erklärungsgrund für die anomale Ablenkung abgeben, oder aber es konnte die Ursache in der beträchtlichen Nenge vou Maguetismus gefunden werden, wclche in dem Manneten nach Unterbrechung des Stromes zurückblieb. 1)iese Vernuthungen bedingten zwei Abänderungen des Versuches.

23. Versuch 16. Waren bei der in achten Versuch benutzten Spirale die Windungen von der Mitle entfernt, so wurde jetzt cine Spirale construirt, deren Windungen umgekchrt sich besonders gegen dic Mitte anbiuften. Im Durchschnit und ron oben gesehen glich diesclbe etwa der Taf. III Fig. 9. Auf cine l'approlle von $95^{\mathrm{mm}}$ Liinge, die an

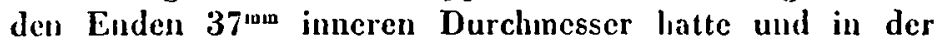
Mitte bis auf $19^{\text {mu }}$ inueren Durchmesser sich verengte, wur- 
den $201^{\text {mtr }}$ mit Seide überspounenen und lackirten Kupferdrahtes, etwa $1^{\mathrm{kgr}}, 3$ schwer, anfgewunden, so dafs die Räume $p$ und $q$ damit crfüllt waren. In der Mitte war ein innen geglättetes Holzröhrchen $c$ eingelassen, durch welches der Faden der aufzuliängenden Substanzen gezogen werden konute. Diese Spirale wurde wie die vorigen zwischen die beiden in der Figur durch punktirte Linien angedeuteten Halbanker $N$ und $S$ des grofsen Magneten gestellt. Die Pfeile $v, \sigma, a$ und $b$ haben dieselbe Bedcutung, wie in der vorigen Figur. Wurden die Versuche mit den in No. 21 und 23 aufgeführten Substanzen wiederholt (dic bisweilen in ihrer Form soweit abgeändert werden mufsten, bis sie sich in der Spirale frei bewegen konnten), so zeigte sich dasselbe Resultat, wie vorher. Die Substanzen der 21. Nummer wurden in der Richtung $a$, die der 23. Nummer in der Richtung $b$ abgelenkt. Noch aufälliger wurde aber das Resultat dadurch, dafs ein Stückchen weicher Eisendraht ( $41^{\mathrm{mm}}$ lang, $3^{\mathrm{mm}}$ Durchmesser, $2^{\mathrm{gr}}, 2$ sehwer) ganz dieselbe Ablenkung erfuhr, wie Wismuth, Wachs, Schwefel u. s. w. Er wurde allerdings von den Polen auch des unthätigen Magneten stark angezogen und stellte sich innerhalb der Spirale diagonal, mit den Enden gegen die Wandungen derselben gelehnt, sprang aber sofort nach der Richtung $a$ herum, sobald die Spirale nach Analogie der Figur erregt wurde, und er sich vorher zufällig mit dem vorderen Ende gegen $N$, mit dem hinteren gegen $S$ gelebnt hatte. Rein magnetische Substanzen verhielten sich also wie diamagnetische, während schwach magnetische Substanzen die entgegengesetzte Ablenkung der vorigen erfuhren.

24. Versuch 17. Es blieb also nur noch übrig, den Grund in dem remanenten Magnetismus des Elektromagneten zu suchen. Der vorige Versuch wurde demgemäfs dahin abgeändert, dafs die beiden Halbanker $N$ und $S$ durch zwei gesonderte, kleinere mit den entgegengesetzten Polen eimander zugewandte Elektromagnete substituirt wurden, bestehend aus zwei Eisenkernen von $165^{\mathrm{mm}}$ Länge und $33^{\mathrm{mm}}$ Durchmesser mit flachgeschliffenen Polen, und erregt 
durch die in No. 19 beschriebenen Spiralen. Der in diesen Magneten zurückbleibende Magnetismus konnte vollständig vernachlässigt werden. Um die Versuche nicht unnöthig zu vervielfaltigen, wurde die Spirale entweder mit 4 (entsprechend einer Ablenkung an der Tangentenbussole von $11^{\frac{3}{4}}{ }^{\circ}$ ) oder mit 10 Platinzinkelementen (entsprechend 25 $\frac{3}{4}^{\circ}$ ) erregt, und ebenso wurden die Elektromagnete durch 10 (entsprechend $42 \frac{1}{2}^{\circ}$ ) oder durch 4 Elemente (entsprechend $22 \frac{1}{4}^{\circ}$ ) in Thitigkeit versetzt. Es wurden nun abermals alle genannten Substanzen geprüft; die in der 21. Nummer aufgeführten bewahrten ihr dort beschriebenes Verhalten, nanentlich wurde Wismuth stets in demselben Sinne abgelenkt, wie ein Eisenstibchen. Von den in der 22. Num-mer aufgeführten Substanzen zeigten aber jetzt

Zink, cin Stäbchen von $41^{\mathrm{mm}}$ Länge, $5^{\mathrm{mm}}$ Durchmesser und $6^{\mathrm{gr}}, 18$ schwer, chemisch rein,

Neusilber, das dort benutzte Parallelepiped,

Platin, ein dreimal zusammengelegter Draht ron $144^{\mathrm{nm}}$

Länge, $l^{\mathrm{mm}}$ Durchmesser und $\mathbf{l}^{\mathrm{gr}}, 8$ Gewicht, meistentheils dasselbe Verhalten, wie Wismuth und Eisen. Für Zink war die Ablenkung im Sinne $a$ besonders deutlich, wenn die Spirale durch 10, die Magnete durch 4 Elemente erregt wurden. Waren an der Spirale 4 , an den Magneten 10 Elemente thätig, dann trat die Ablenkung $a$ erst ein, wenn vorher der Strom der Magnete für einen Moment geöffnet war. Dasselbe fand beim Platin statt. Dagegen aber bewalurte

Ordinäres braunes Siegellack $\left(41^{\mathrm{mm}}\right.$ lang, $11^{\mathrm{mm}}$ brcit, $6^{\mathrm{mm}}$ dick und $3^{* r}, 6$ schwer, in welchem durch chemische Untersuchung ein sehr beträchtlicher Gehalt an Eisenoxyd nachgewiesen wurde) und cine

Neusilberplatte $\left(3^{\text {mm }}\right.$ lang, $13^{\text {mm }}$ breit, $2^{\text {mun }}$ dick und $8^{8 r}, 5$ schwer, deren kürzeste Abmessung dem Aufhängefaden und deren längste der Axe der Spirale parallel war )

noch inmer die durch die Richtung des Pfeiles $b$ angegebene Ablenkung. Doch bald zeigte sich, dals dieses nur 
der Fall war, wenn die Magnete früher in Thätigkeit versetzt zourden, als die Spirale. Wurde aber die Spirale zuerst erregt, und etwa nach $\frac{1}{2}$ Minute der Strom der Magnete geschlossen, dann zeigten auch diese Substanzen die Ablenkung $a$. Das Siegellack konnte sogar, wenn die Magnete nur einmal in Thätigkeit gewesen waren, durch die Spirale allein, oder aber durch die Magnete allein in der einmal thätig gewesenen Spirale willkührlich abgelenkt werden, je nachdem der Strom die eine oder andere Richtung erhielt. Hieraus geht aber hervor, dafs

Substanzen wie Neusilber, Zink, Platin, Siegellack ( das mit Eisenoxyd braun gefärbt ist) u. s. w., eine ähnliche und vielleicht noch gröfsere Coërcitivkraft (d. i. ein Vermögen den einmal angenommenen Magnetismus zu bewahren) besitzen wie der Stahl; dafs diese Coërcitivkraft nur einen Moment zu täuschen vermochte, dafs aber diese genannten Körper durch Spirale und Magnete dieselbe normale Polarität erfahren, wie Wismuth und Eisen.

In Wahrheit verhielt sich ein in der thätigen Spirale bei unthätigen Magneten aufgehangener Transversalmagnet, dargestellt aus einer platten Feile von $6^{\mathrm{mm}}$ Länge, $8^{\mathrm{mm}}$ Breite und kaum $\frac{1}{2}^{\mathrm{mm}}$ Dicke ganz so wie das Siegellack oder das Neusilber. Da namentlich gemengte Substanzen diese eigenthümliche Coërcitivkraft zeigen, versuchte ich, ob dieselbe auch bei einer Platte von Rose'schem Metall nachzuweisen wäre. Doch verhielt sich dieselbe ganz so, nur schwächer, wie Wisınuth.

25. Eine Analogie der hicr dargestellten Erscheinungen bietet wohl das ron Hrn. Plücker beobachtete Verhalten eines Cyanitkrystalles, der sich schon in Folge des Erdmagnetismus von Nord nach Sïd stellte, aber zwischen den Magnetpolen seine permanente Polarität verlor '). Im. gleichen verblieben Eisenglanzkrystalle von Elba ${ }^{2}$ ), Augit und Zinnstein in jeder Lage zwischen den Magnetpolen,

1) Pogg. Ann. Bd. 77, S. 447.

2) Pogg. Ann. Bd. 78, S. 421. 
wenn sic cine Weile in dersclben festgehalten worden waren.

26. Dafs insbesondere bei gemengten Substanzen, wie Neusilber und Siegellack, die Coërcitivkraft, so deutlich auftritt, scheint neue Belege zu den Beobachtungen des Hrn. Z a d d a ch in dessen sorgfältigen Untersuchungen " über die inagnetische Polarität des Basaltes und der trachytischen Gesteine “') zu liefern. Hr. $Z$ a dda ch führt nämlich durch, dafs die Coërcitivkraft namentlich dann hervortritt, wenn magnetische Substanzen in zerklüftetem oder verwittertem Zustand (wic gerostetes Eisen, Basaltstücke die lange Zeit der Wirkung der Atmosphäre ausgesetzt waren) sich befinden, oder wenn sie mit minder magnetischen Substanzen innig gemengt sind (wie Eisenoxyd mit Oxydul im Magneteisenstein, Eisen mit Kobleeisen im Stahl, Magueteisen mit Augit und Feldspath im Basalt u. s. w.).

27. Vor allem geht aber aus den Untersuchungen der 20. bis 24. Numıner hervor:

dafs die kleinsten Theilchen sowohl der eisenmagnetischen, als der wismuthmagnetischen Substanzen dicselbe normale Polarität erhalten, wenn sie der magnetischen Scheidungskraft ciner galvanischen Spirale ausgesetzt werden.

Denn nur durch diese Annahme lassen sich alle beobachteten Thatsachen ungezwungen und ohne Herbeiziehen anderer Hypothesen erklären.

28. Aus den Gesammtuntersuchungen dieser AbtheiIung bin ich aber gezwungen, gegenüber einer gewichtigen Autorität, zu wiederbolen:

Dafs die von Hrn. We b e $r$ in scinen "elektrodyuamischen Mafsbestimmungen, insbesondere über Diamagnetismus " zu Grunde gelegten Versuche zwar bestätigt worden sind, aber anders erklärt werden müssen, als es dort gescliah.

Dic

1) Bunn bei G corgi 185̄l. Auszugsweise in "Künigsberger naturwisscnschaftliche Unterhaltungen" Bd. 2, Heft 3. 
Die dort gefundene Ablenkung eincs Magneten durch einen in einer galvauischen Spirale auf- und abbewegten Wismuthstab rührt wenigstens zum überwiegend gröfsten Theil von Inductionsströmen her, die durcb die mechanische Bewegung innerhalb der Spirale im Wismuth erregt werden.

Die noch etwa übrig bleibende anormale Polarität des Wismuth erklärt sich durch die gewählte eigenthümliche Form der Spirale.

Die durch erregtes Wismuth von Hrn. Weber gefundenen Inductionsströme in einem geschlossenen Leiter sind demnach secundäre Inductionsströıne.

Es ist also nicht bewiesen, dafs die Polarität der kleinsten Theilchen in Fismuthmagnetischen Substanzen bei gleicher idealer Vertheilung des Magnetismus die umgekehrte sey, als die in eisenmagnetischen.

Wird dagegen ein Wismuthstab, von der Mitte seiner Längsausdehnung her, stärker erregt, als von seinen Enden her, alsdann zeigt er bei gleichgerichteter magnetischer Scheidungskraft eine gleichgerichtete (und ziemlich starke) ideale Vertheilung des Magnetismus als das Eisen.

Deıngemäfs ist die Polarität der kleinsten Theilchen wismuthnagnetischer Körper bei gleichgerichteter magnetischer Scheidungskraft dieselbe, als dic der kleinsten Theilchen eisenmagnetischer Körper.

(Schluls im nächsten Heft.)

Poggendorff's Annal. Bd. XCII. 